\title{
Propriedades funcionais da farinha do mesocarpo do coco babaçu: uma alternativa nutricional contra a Covid-19
}

Functional properties of babassu coconut mesocarp flour: a nutritional alternative against Covid19

Propiedades funcionales de la harina de mesocarpio de coco babasú: una alternativa nutricional

frente al Covid-19

Recebido: 10/02/2021 | Revisado: 16/02/2021 | Aceito: 22/02/2021 | Publicado: 28/02/2021

Kaio Germano Sousa da Silva

ORCID: https://orcid.org/0000-0003-4236-6230

Centro Universitário de Ciências e Tecnologia do Maranhão, Brasil

E-mail: kaiogsds@hotmail.com

Karine Costa Melo

ORCID: https://orcid.org/0000-0001-8253-859X

Centro Universitário de Ciências e Tecnologia do Maranhão, Brasil

E-mail: Karinemelo09@gmail.com

Maria Eduarda Leal de Carvalho Santos

ORCID: https://orcid.org/0000-0003-3400-0570

Centro Universitário de Ciências e Tecnologia do Maranhão, Brasil

E-mail: eduardalealcs@gmail.com

Atalia Talita da Silva

ORCID: https://orcid.org/0000-0003-3726-685X

Centro Universitário de Ciências e Tecnologia do Maranhão, Brasil

E-mail: talitaatalia877@gmail.com

Alanna Sanlai Sousa Lima

ORCID: https://orcid.org/0000-0001-8026-5743

Centro Universitário de Ciências e Tecnologia do Maranhão, Brasil

E-mail: alanasanlaif2@gmail.com

Natália Monteiro Pessoa

ORCID: https://orcid.org/0000-0001-6502-5661

Centro Universitário de Ciências e Tecnologia do Maranhão, Brasil

E-mail: natalia_tulip@hotmail.com

Letícia Aparecida Santos Silva

ORCID: https://orcid.org/0000-0002-8000-7071

Centro Universitário de Ciências e Tecnologia do Maranhão, Brasil

E-mail: leticia.odonto01@ outlook.com

Liejy Agnes dos Santos Raposo Landim

ORCID: https://orcid.org/0000-0002-8214-2832

Universidade Federal do Piauí, Brasil

E-mail: liejyagnes@gmail.com

Ana Paula de Melo Simplício

ORCID: https://orcid.org/0000-0002-0059-9231

Universidade Federal do Piauí, Brasil

E-mail: anapaulamsim@hotmail.com

Fernanda de Oliveira Gomes

ORCID: https://orcid.org/0000-0002-8763-0448

Universidade Federal do Piauí, Brasil

E-mail: fernanda.oliveira.sa31@gmail.com

Janaina Oliveira Morais

ORCID: https://orcid.org/0000-0002-4315-2691

Centro Universitário de Ciências e Tecnologia do Maranhão, Brasil E-mail: oliveira.janana@ hotmail.com

Francisca Thawanny de Souza Silva

ORCID: https://orcid.org/0000-0003-2880-0985

Centro Universitário de Ciências e Tecnologia do Maranhão, Brasil

E-mail: kaiogermano123890@gmail.com

Elis Regina Oliveira Morais

ORCID: https://orcid.org/0000-0002-1248-9929

Universidade Estadual do Maranhão, Brasil

E-mail: morais-elis@bol.com.br 
Telvanise Oliveira Morais

ORCID: https://orcid.org/0000-0001-8286-0466 Faculdade do Vale do Itapecuru, Brasil E-mail: tnise2009@hotmail.com

\begin{abstract}
Resumo
Com o avanço da pandemia do patógeno Coronavírus SARS-CoV-2, causador da doença Covid-19 (Coronavirus Disease), sendo considerada hoje uma grande ameaça em escala global, recomenda-se uma alimentação rica em propriedades funcionais no combate e na prevenção dos agravos de sintomatologia dessa patologia. Dessa forma, a pesquisa traz como objetivo apresentar a importância das propriedades funcionais do coco babaçu no aporte imunológico e no combate a síndrome inflamatória causada pela Covid-19. Trata-se de uma revisão bibliográfica descritiva com abordagem qualitativa da literatura científica, compreendendo o período de 2010 a janeiro de 2021. Como resultado, o aporte nutricional com propriedades funcionais melhora a função imune e auxilia no enfretamento de inflamações, reduzindo a gravidade de doenças inflamatórias. Desta forma, controlar a inflamação é uma estratégia efetiva para reduzir a gravidade da Covid-19, e a farinha do mesocarpo do coco babaçu é uma opção de suplementação no combate a processos inflamatórios justamente por se tratar de um alimento antioxidante e antiinflamatório natural. Conclui-se que a farinha do mesocarpo do coco babaçu por se constituir como um complemento alimentar altamente nutritivo e com uma grande capacidade para tratar diversas patologias, justifica-se seu provável potencial para tratar a sintomatologia da Covid-19, incluindo ainda a Síndrome Respiratória Aguda Grave, pois sua composição é formada por diversas vitaminas e minerais, tornando-o um alimento profundamente anti-inflamatório e antioxidante.
\end{abstract}

Palavras-chave: Babaçu; Alimento funcional; Infecções por coronavírus.

\begin{abstract}
With the advance of the pandemic of the pathogen Coronavirus SARS-CoV-2, which causes the disease Covid-19 (Coronavirus Disease), being considered today a great threat on a global scale, a diet rich in functional properties in the combat and prevention of diseases is recommended. symptoms of this pathology. Thus, the research aims to present the importance of the functional properties of babassu coconut in immunological support and in combating the inflammatory syndrome caused by Covid-19. This is a descriptive bibliographic review with a qualitative approach to the scientific literature, covering the period from 2010 to January 2021. As a result, the nutritional supply with functional properties improves the immune function and helps to cope with inflammation, reducing the severity of diseases inflammatory. Thus, controlling inflammation is an effective strategy to reduce the severity of Covid-19, and babaçu coconut mesocarp flour is a supplementation option to combat inflammatory processes precisely because it is a natural antioxidant and anti-inflammatory food. . It is concluded that the Babaçu coconut mesocarp flour constitutes a highly nutritious food supplement and with a great capacity to treat various pathologies, its probable potential for treating the symptoms of Covid-19, including the Respiratory Syndrome, is justified Acute Grave, because its composition is formed by several vitamins and minerals, making it a deeply anti-inflammatory and antioxidant food.
\end{abstract}

Keywords: Babaçu; Functional food; Coronavirus infections.

\title{
Resumen
}

Con el avance de la pandemia del patógeno Coronavirus SARS-CoV-2, que provoca la enfermedad Covid-19 (Enfermedad del Coronavirus), siendo considerada hoy una gran amenaza a escala mundial, se recomienda una dieta rica en propiedades funcionales en el combate y prevención del agravo de los síntomas de esta patologia. Así, la investigación tiene como objetivo presentar la importancia de las propiedades funcionales del coco babasú en el apoyo inmunológico y en la lucha contra el síndrome inflamatorio causado por el Covid-19. Se trata de una revisión bibliográfica descriptiva con un enfoque cualitativo de la literatura científica, que abarca el período de 2010 a enero de 2021. Como resultado, el aporte nutricional con propiedades funcionales mejora la función inmunológica y ayuda a afrontar la inflamación, reduciendo la gravedad de las enfermedades inflamatorias. Así, controlar la inflamación es una estrategia eficaz para reducir la severidad del Covid-19, y la harina de mesocarpio de coco de Babaçu es una opción de suplementación para combatir los procesos inflamatorios precisamente por ser un alimento antioxidante y antiinflamatorio natural. Se concluye que la harina de mesocarpio de coco de babaçu constituye un complemento alimenticio altamente nutritivo y con una gran capacidad para tratar diversas patologías, su probable potencial para tratar los síntomas de Covid-19, incluido el Síndrome Respiratorio Agudo Grave, se justifica debido a su composición está formado por varias vitaminas y minerales, lo que lo convierte en un alimento profundamente antiinflamatorio y antioxidante.

Palabras clave: Babaçu; Alimentos funcionales; Infecciones por coronavirus. 


\section{Introdução}

Com o avanço da pandemia do patógeno Coronavírus SARS-CoV-2, causador da doença Covid-19 (Coronavirus Disease), sendo considerada hoje uma grande ameaça em escala global. Mudou-se drasticamente o comportamento da população, devido à necessidade de distanciamento social aconselhado pela Organização Mundial de Saúde (OMS), a fim de retardar a disseminação da doença (Oliveira, Abranches, \& Lana, 2020).

Desta forma, uma das complicações causada pela Covid-19 é a síndrome inflamatória ocasionada pelo patógeno SARS-CoV-2. Tendo como causa uma diminuição ligeiramente considerável de linfócitos, como resultado deste declínio, há uma queda na imunidade, deixando o organismo sem suas defesas naturais, tornando o corpo do hospedeiro aberto a infecções e complicações do novo Coronavírus (Organização Mundial de Saúde [OMS], 2020).

Sendo assim, com as restrições propostas pela OMS no que refere ao isolamento social, a população procurou fortalecer o sistema imunológico com fitoterapia e alimentos com propriedades funcionais, mas em contrapartida a ingestão de processados e ultraprocessados aumentaram drasticamente, o que contribuiu para incidência e descompensamento de doenças crônicas em pessoas já acometidas, onde uma alimentação desta natureza contribui fortemente para o estresse oxidativo no organismo. Em razão disso, percebe-se a importância de uma ingestão de alimentos saudáveis aliados com hábitos e estilo de vida corretos no enfretamento da pandemia de Covid-19 (Conselho Federal de Nutricionistas [CFN], 2020).

Desta forma, recomenda-se uma alimentação rica em propriedades funcionais no combate e na prevenção dos agravos de sintomatologia da Covid-19. Neste sentido, vitaminas e minerais vem sendo discutidos e cogitados, uma vez que alguns nutrientes têm um grande potencial de facilitar e modular a resposta imune do indivíduo acometido pela Covid-19, a fim de minimizar consequências e o avanço da patologia, suavizando assim as manifestações clínicas e consequentemente favorecendo as intervenções e cooperando na melhora do paciente e qualidade de vida do mesmo (Lima et al., 2020).

Destaca-se a farinha do mesocarpo do babaçu (FMB), conhecida popularmente pelas suas propriedades antiinflamatórias e analgésicas, e usada no tratamento de várias patologias, entre elas doenças imunossupressoras (Silva et al., 2017). Além disso, é um alimento rico em sais minerais, vitaminas e amido, podendo ser utilizado para panificação. O aproveitamento integral desta matéria-prima proporciona ainda, uma fonte de renda para a população, e insere a FMB em biscoitos na suplementação da alimentação com o intuito de prevenir doenças ou combatê-las (Melo et al., 2007).

O presente estudo teve como pergunta norteadora "qual a importância da farinha do mesocarpo do coco babaçu na prevenção de complicações causadas pelo novo coronavírus?". Como objetivo, busca apresentar a importância das propriedades funcionais do coco babaçu no aporte imunológico e no combate a síndrome inflamatória causada pela Covid-19.

\section{Metodologia}

O presente estudo consiste em uma revisão bibliográfica descritiva com abordagem qualitativa da literatura científica, compreendendo o período de 2010 a janeiro de 2021. Para o levantamento dos artigos foi realizada uma busca nas seguintes bases de dados: Literatura Latino-Americana e do Caribe em Ciências da Saúde (LILACS), Medical Literature Analysis and Retrieval Sistem on-line (Medline, via Pubmed) e Scientifc Electronic Libray Online (SCIELO) e Google Acadêmico.

Foram utilizados para busca dos artigos os seguintes descritores e suas combinações nas línguas portuguesa, espanhola e inglesa: "babaçu 1", "propriedades funcionais 2", "Covid-19 3". Termos semelhantes foram usados nas outras bases de dados. Foram localizados 30 artigos de acordo com esses termos. Em seguida, fez-se a leitura minuciosa, resultando em 15 artigos, sendo 5 artigos oriundos do Google acadêmico, 8 oriundos da Scielo e 2 oriundos da Pubmed.

Foram avaliados para critério de inclusão: identificação do artigo, ano de publicação no recorte temporal (2010 a 2021) e propriedades funcionais da farinha do mesocarpo do coco babaçu mencionados em auxiliar no embate da síndrome 
inflamatória causada pelo Covid-19. Também se pesquisou documentos governamentais e foram incluídos os trabalhos que relacionaram diretamente os efeitos benéficos das propriedades funcionais da farinha do mesocarpo do coco babaçu.

Foram excluídos artigos em forma de cartas ao editor, comentários, série de casos, resumos não publicados, trabalhos que não se adequavam ao tema proposto em conjunto com os descritores "1", "2", "3"; aqueles publicados em um recorte temporal inferior a 2009, e os que não se apresentavam disponíveis em textos completos. Os artigos foram lidos de forma minuciosa e crítica, e selecionados os textos que melhor abordaram o tema.

\section{Resultados e Discussão}

\subsection{Coronavirus (Covid-19) X Nutrição: alimentação e estilo de vida saudável, nutrientes e alimentos funcionais}

No ano de 2019, o mundo se deparou com o surgimento de um novo vírus nomeado de SARS-coV-2, esse vírus apareceu pela primeira vez na China e tornou-se responsável pelo surgimento da doença Covid-19. Rapidamente, já no ano de 2020, novo vírus se disseminou em vários países, o que fez com que em 11 de março de 2020 a OMS - Organização Mundial de Saúde elevasse o estado da contaminação à Pandemia (Associação Brasileira de Nutrição [ASBRAN], 2020).

O Brasil registrou o primeiro caso de covid-19 em fevereiro de 2020, após 06 meses do primeiro caso o país ultrapassou o número de mais de 100 mil mortes. Devido ao altíssimo número de mortos, as autoridades sanitárias brasileiras instituíram normas em relação a velórios e sepultamentos durante o período da pandemia, a vítima de Covid-19 foi classificada como agente biológico risco 3 (OMS, 2020).

Trazendo para o contexto da alimentação, o isolamento social tem influência na compra e consumo de alimentos industrializados. Na tentativa de reverter quadros de possíveis carências nutricionais na pandemia, foi identificada a procura de alimentos ricos em propriedades funcionais, porém a população tende a comprar mais alimentos processados e/ou ultraprocessados, pois os mesmos tornam-se mais práticos, menos perecíveis e possuem valores mais acessíveis. No entanto, obter uma dieta balanceada é essencial para lidar com essa doença, portanto, a segurança alimentar deve ser uma das questões atuais de saúde e higiene (Oliveira et al., 2020).

No que se refere a alimentação saudável, tem-se como característica principal aquela que atende todas as necessidades nutricionais do corpo. Além de ser uma fonte de nutrientes, a alimentação dita saudável envolve aspectos, entre eles sociais, culturais, sensoriais e afetivos. Uma alimentação deve ser variada, equilibrada em quantidade e acesso, colorida e segura, dando prazer e identidade cultural e familiar, podendo prevenir o aparecimento de doenças, sendo essencial para promover e manter a saúde (Organização Pan-Americana da Saúde [OPAS], 2019).

Segundo Cardoso et al. (2016), o estilo de vida inadequado pode colaborar negativamente para a manifestação precoce de algumas doenças, assim como a adoção de práticas saudáveis, de forma cotidiana, pode contribuir para melhoria da saúde humana bem como prevenir contra possíveis patologias. Uma alimentação equilibrada em consonância com outros fatores contribui fortemente para uma resposta no âmbito imunológico satisfatório.

Alguns parâmetros são indispensáveis para conservação de um estilo de vida saudável, são eles: controle do estresse, prática de atividade física regular, bons relacionamentos sociais e hábitos alimentares saudáveis. Portanto, a realização de atividade física associada a uma alimentação variada e equilibrada contribui positivamente para um indivíduo que busca um estilo de vida saudável, contudo, vale ressaltar que devido às diversas modificações ocorridas no cotidiano, os hábitos saudáveis algumas vezes ficam em segundo plano (Souza et al., 2016).

Atualmente, dados disponíveis na literatura científica apontam que o estilo de vida de um indivíduo influencia diretamente na qualidade de vida do mesmo, de forma positiva ou não. Práticas comportamentais como o uso de medicamentos, realização de atividade física, tabagismo, alcoolismo, controle clinico de doenças e a ingestão alimentar são 
fatores usados para ações de estratégias de prevenção de agravos a saúde (Levy, Claro, Bandoni, Mondini, \& Monteiro, 2012; Zaitune et al., 2012).

Desta forma, ingestão desequilibrada de nutrientes associada ao sedentarismo colabora negativamente para alteração no perfil antropométrico da pessoa. Estudos demostram que a ingestão de industrializados diminui a absorção de vitaminas e minerais e com isso ocasiona um declínio do aporte imunológico, bem como eleva a quantidade de gordura abdominal, aumentando o risco de doenças crônicas, onde essas são consideradas fatores de risco de complicações da covid-19 (OMS, 2020).

Sendo assim, a qualidade nutricional é positiva no que se refere à determinação de um estado de saúde longevo, pautado em uma compreensão da relevância de uma alimentação com qualidade e quantidade, que é transformada com o tempo, tendo influência de fatores sociais e econômicos, que interagem e que integram uma forma de viver mais saudável (OPAS, 2019).

Com isso, uma maior ingesta alimentar de nutrientes engloba todas as fases e aspectos da vida, e vem a ser de grande importância neste período de pandemia. Alimentos funcionais com vitamina D, C, E, ferro, cálcio, magnésio, fosforo entre outros, já são comprovados cientificamente de sua importância no combate a Covid-19, haja vista que a busca por alimentos com propriedades funcionais e chás medicinais aumentou muito neste período, com a necessidade de melhorar a resposta imune, já que a alimentação saudável proporciona a manutenção da saúde e o fortalecimento do sistema imunológico (ASBRAN, 2020).

É vista a importância de alimentos funcionais ricos em vitaminas e minerais, com taxa de biodisponibilidade rápida no combate a síndrome inflamatória causada pelo Coronavírus SARS-CoV-2, onde estas complicações causam um declínio rápido de linfócitos T comprometendo a imunidade, deixando o organismo suscetível a infecções. Portanto, uma boa alimentação com propriedades funcionais pode ajudar no combate e na prevenção dos agravos de sintomatologia da covid-19 (Lima et al., 2020).

Neste aspecto, a farinha do mesocarpo do coco babaçu é uma importante complementação alimentar devido ao seu grande valor nutricional. Sendo utilizada em tratamentos de reumatismo, artrite, úlcera, tumores e inflamações em gerais (útero, ovários), também é muito indicada em prisão de ventre, colite e obesidade para auxiliar no emagrecimento, um antiinflamatório natural e rico em micronutrientes, macronutrientes e minerais (Baldez et al., 2017).

\subsection{Babaçu}

O babaçu em suas características é um tipo de planta que faz parte da vasta família de arececae do tipo palmeira (Orbignya Speciosa). Existem muitas variedades do coco babaçu, dentre as mais conhecidas e de uso variado e difundido estão: Attlea, Phalerata e Attlea Spciosa. A nomenclatura do babaçu vem de origem Tupi-Guarani, sendo que Ba tem significado de fruto e Açu significa grande (Silva et al., 2012).

Deste modo a palmeira do coco babaçu tem sua origem dentro do território brasileiro tendo uma grande extensão geográfica nas regiões Norte, Nordeste e Centro-Oeste, e uma maior prevalência no Nordeste. Estima-se que existam em torno de 18 a 20 milhões de hectares com palmeiras de coco babaçu dentro do Brasil, sendo que metade dessa área está localizada no estado do Maranhão, estado esse que tem o babaçu como fonte econômica de famílias e também como fonte cultural, pois além de ser a terra do bumba-meu-boi, dos lençóis de águas cristalinas e deltas, é conhecida também como terra do babaçu (Pinto, Amaral, Gaia, \& Oliveira 2014).

No que se refere no âmbito econômico, o babaçu (Orbignya Speciosa) possui um grande valor socioeconômico, com sua maior utilização nos estados do Maranhão, Piauí, Tocantins, Mato Grosso, Goiás, Amazonas, Pará, Rondônia, Ceará, Bahia e Minas Gerais, sendo que tal fruto tem plantio em 279 municípios, onde seu extrativismo é muito forte e gera ocupação para milhares de famílias (Ministério do Desenvolvimento Social e Combate à Fome [MDS], 2019). 
Dentro do aspecto de plantação, o babaçu (Orbignya Speciosa) ocorre de forma espontânea em planícies e áreas de baixa declividade, com adaptações em vários tipos de solo e climas. A plantação de babaçu tem pioneirismo em áreas abertas como, por exemplo, pastagens, onde a ocorrência de uma área vasta de babaçus diminui a predominância de floresta fechada. Possui registros de florestas de babaçus em vários países como Suriname, Peru e Brasil (Silva, 2011).

Este fruto tem uma vasta utilização que vai desde a geração de energia ao artesanato, diversas atividades econômicas podem ser feitas através da planta. Dentre as partes da planta, o fruto tem o maior potencial econômico para aproveitamento tecnológico e industrial, podendo produzir aproximadamente 64 produtos, tais como carvão, etanol, metanol, farináceos, celulose, ácidos graxos, glicerina, porém basicamente são produzidos apenas o carvão e o óleo em escala industrial. O babaçu aliado aos demais produtos fornece ao homem do campo um meio de sobrevivência que contribui na renda das famílias, fixando os trabalhadores do campo e promovendo o desenvolvimento da agroindústria no meio rural (ASBRAN, 2020).

\subsection{Propriedades funcionais da farinha do mesocarpo do coco babaçu como alternativa nutricional no aporte imunológico e no combate ao Covid-19 em tempos de pandemia}

A ocorrência de óbitos relacionados à Síndrome Respiratória Aguda Grave causada pelo Coronavírus (SARS-CoV-2) está mais presente no grupo de idosos, onde, muitos destes indivíduos apresenta comorbidades (imunossupressão e obesidade). Destacam-se ainda pacientes acometidos por câncer, que também estão em imunossupressão e sensíveis a grandes riscos de complicações graves infecciosas por Covid-19 (European Food Safety Authority [EFESA], 2020).

Desta forma, pacientes com comorbidades estão predispostos à imunossupressão e a chamada síndrome inflamatória, o que infelizmente, contribui para mortalidade, onde o aporte nutricional com propriedades funcionais melhora a função imune e auxilia no enfretamento de inflamações, reduzindo a gravidade de doenças inflamatórias. Desta forma, controlar a inflamação é uma estratégia efetiva para reduzir a gravidade da Covid-19 e a farinha do mesocarpo é uma opção de suplementação no combate a processos inflamatórios justamente por se tratar de um alimento antioxidante e anti-inflamatório natural (Grant et al., 2020; Silva et al., 2017; Morais, Maia, Damasceno, Seabra, \& Passos, 2020).

Desta forma, o mesocarpo do babaçu é bem usado no Brasil em terapias populares, seja ela em suplemento alimentar ou na medicina empírica. A farinha é muito utilizada para o tratamento de inflamações, onde age combatendo doenças que desencadeiam inflamações, podendo vir a ser útil contra Covid-19, cólicas menstruais e até leucemia, devendo-se testar ainda mais seus possíveis benefícios. Além de suas amêndoas, que contém uma substância que produz óleo ou azeite, importante terapeuticamente e na cocção de alimentos, as folhas também são utilizadas em tinturas e chás (MDS, 2019).

A farinha do babaçu ou também pó do babaçu é $100 \%$ natural caracterizando cerca de 17 a $22 \%$ do fruto e também $60 \%$ de amido, apresenta na sua composição $20 \%$ de fibras, 8 a $15 \%$ de umidade e 4 a $5 \%$ de outras substancias, como sais minerais, vitaminais taninas e uma pequena quantidade de proteínas. Onde um estudo feito pela China Medical University (2021) localizada em Taiwan, descobriu que o tanino presente nos vinhos tintos tem o poder de inibir duas enzimas-chaves do vírus, prevenindo a infecção e controlando o aumento da carga viral, tal pesquisa tinha o objetivo de encontrar compostos naturais capazes de combater efeitos e complicações da Sars-CoV-2. O ácido tânico, substância pertencente a classe dos taninos, já tem suas propriedades funcionais comprovando sua eficiência contra a SARS em 2003 (Khalifa, Zhu, Mohammed, Dutta, \& Li, 2020).

Os taninos são substancias antioxidantes e anti-inflamatórios (Melo et al., 2017). Devido a uma grande presença de taninos na farinha do mesocarpo do coco babaçu, o pó tem a coloração castanha (Almeida et al., 2011).

Estudos demostram uma vasta composição da farinha do mesocarpo do babaçu, onde a mesma apresenta valores consideráveis de fibras, cálcio, magnésio, fósforo, ferro, zinco, vitamina D e outros nutrientes importantes para manutenção da 
saúde, segundo valores extraídos da Tabela de Composição de Alimentos (TACO) do Núcleo de Estudos e pesquisas em Alimentação (NEPA) (2011), descritos abaixo na Quadro 1.

Quadro 1. Composição química e valor energético da farinha do mesocarpo do babaçu, utilizando o valor de referência de $100 \mathrm{~g}$ da farinha.

\begin{tabular}{|l|c|}
\hline COMPOSTOS & COMPOSIÇÃO \\
\hline Energia & $329 \mathrm{Kcal}$ \\
\hline Carboidratos & $79.2 \mathrm{~g}$ \\
\hline Lipídeos & $0.2 \mathrm{~g}$ \\
\hline Proteína & $1,4 \mathrm{~g}$ \\
\hline Colesterol & $\mathrm{NA} \mathrm{g}$ \\
\hline Ferro & $18.3 \mathrm{~g}$ \\
\hline Cálcio & $61 \mathrm{~g}$ \\
\hline Vitamina D & $300,000 \mathrm{UI}$ \\
\hline Vitamina E & $2,500 \mathrm{mg}$ \\
\hline Vitamina K3 & $300 \mathrm{mg}$ \\
\hline Vitamina B1 & $380 \mathrm{mg}$ \\
\hline Vitamina B2 & $1000 \mathrm{mg}$ \\
\hline Vitamina B12 & $2000 \mathrm{mg}$ \\
\hline Ácido Fólico & $162,50 \mathrm{mg}$ \\
\hline Magnésio & $39 \mathrm{~g}$ \\
\hline Vitamina A & $140,000 \mathrm{UI}$ \\
\hline Zinco & $17,500 \mathrm{mg}$ \\
\hline Cinzas & $3,4 \mathrm{~g}$ \\
\hline
\end{tabular}

Fonte: NEPA (2011).

Como se nota, a farinha do mesocarpo do coco babaçu possui em sua constituição importantes substâncias químicas, tais como: triterpenos, taninos, açúcares, saponinas e demais compostos esteroides. Ainda destacam-se seus polissacarídeos que, como já dito anteriormente, são anti-inflamatórios e imunomoduladores importantes no combate à síndrome inflamatória, podendo incluir a inflamação causada pela Covid-19. É observado que o babaçu é um bom cicatrizante, protetor gástrico, antitrombótico e antimicrobiano (Cavalcante Neto, 2012).

A incidência de mortes causadas por Covid-19 são na sua maioria decorrentes de infecções no trato respiratório, onde estas estão associadas aos quadros clínicos de pneumonias e inflamações pulmonares, gerando infecções e a ativação do sistema imune do hospedeiro, de maneira que o resultado muitas vezes, não pode ser controlado (Garófolo, Qiao, Maia-Lemos, 2019).

Por este motivo, o uso de suplementação de vitaminas D, C, E, A, zinco, e ferro vem sendo discutido e cogitado, uma vez que, estes nutrientes têm um grande potencial de facilitar e modular a resposta imune do indivíduo acometido, tendo como resultado positivo destas intervenções a redução da tempestade de citocinas, principalmente na Covid-19. A farinha do mesocarpo do coco babaçu possui em sua composição tais nutrientes, demonstrando assim, sua relevância no ponto de vista nutricional e potencial em criar resposta de defesa contra o novo coronavírus (Grant et al., 2020).

Nesse aspecto, a vitamina D (presente na farinha do babaçu em quantidade considerável), gera diversos benefícios para o corpo humano, pois possui o efeito de modulação da resposta imune. Por isso, o uso desta vitamina no combate as infecções respiratórias, como a gripe pelo vírus Influenza e a infecção pelo Coronavírus 2019, tem sido palco de discussões (Garófolo et al., 2020).

No que refere à imunidade celular, ou seja, dentro da célula a vitamina $\mathrm{D}$, ocorre o aumento da secreção de peptídeos antimicrobianos, reduzindo a tempestade de citocinas. Na imunidade adaptativa, a vitamina D faz a modulação da resposta, com intuito de aumentar o perfil Th2 (anti-inflamatório) e reduzir o perfil Th1 (pró-inflamatório). Com isso, a vitamina D 
permite a instalação de um controle da inflamação para evitar o agravamento de lesões teciduais. Há a necessidade de mais estudos a fim de determinar a concentração para uso. Ressaltando ainda que a Farinha do mesocarpo do coco babaçu é uma fonte deste nutriente e mostra mais uma vez, o poder nutritivo deste alimento naturalmente brasileiro (Silva et al., 2017).

O extrato etanólico da planta, testado em linhagens de células humanas leucêmicas, tumores de próstata e câncer de mama, promoveu diminuição da viabilidade em todas estas com dose-dependente. Neste estudo, o efeito foi mais pronunciado sobre as linhagens de celulares tumorais quando comparado às não tumorais. Além disso, possui atividade antioxidante e capacidade de eliminar os radicais livres, protegendo as células do estresse oxidativo. Além de prevenir o câncer, esse efeito também pode retardar o envelhecimento precoce (Oliveira \& Reyes, 1990).

Segundo o estudo de Barros (2011) foi possível verificar a viabilidade do pó do babaçu em capsulas utilizado por via oral, foram preparados lotes das capsulas de dicoflenaco de sódio de $50 \mathrm{mg}$ e paracetamol de $500 \mathrm{mg}$. O primeiro lote, com a presença do mesocarpo e no outro celulose microcristalina, observou-se que o pó apresentou um resultado satisfatório comparado à celulose, apresentando o perfil de liberação ligeiramente superior. Desta forma, nota-se a importância da farinha do babaçu em facilitar a absorção e transformação de forma rápida dos nutrientes no organismo, fator esse, de grande relevância no combate a Covid-19, onde o tempo é crucial para salvar vidas.

\section{Considerações Finais}

Este estudo permitiu observar a importância das propriedades funcionais do coco babaçu no aporte imunológico, onde a farinha do mesocarpo do coco babaçu pode ser importante no combate à síndrome inflamatória causada pela Covid-19, justamente por se tratar de um alimento que é antioxidante e anti-inflamatório.

Diante dos problemas enfrentados em escala global, buscar alternativas para cessar ou minimizar os agravos ocasionados pelo Coronavírus é a prioridade no momento. Neste aspecto, revela-se a importância de uma vida saudável e uma alimentação balanceada para a prevenção da doença ou ainda, a utilização de alimentos funcionais, como a farinha do mesocarpo do coco babaçu para tratar a inflamação ocasionada pela patologia.

Já que a farinha do mesocarpo do coco babaçu se constitui como um complemento alimentar altamente nutritivo e com uma grande capacidade para tratar diversas patologias, justifica-se seu provável potencial para tratar a sintomatologia da Covid-19 incluindo ainda, a Síndrome Respiratória Aguda Grave, pois sua composição é formada por diversas vitaminas e minerais tornando-a um alimento profundamente anti-inflamatório e antioxidante.

Como limitações da pesquisa tem-se o fato de que, não existem disponíveis na Literatura, muitos artigos comprovando a eficácia da farinha do mesocarpo do coco babaçu como coadjuvante ou ainda produto central no tratamento da sintomatologia da Covid-19.

Por fim, o estudo possibilitou expandir os conhecimentos acerca da importância da alimentação equilibrada, e da possível utilização da farinha do mesocarpo do coco babaçu para o tratamento eficaz da Covid-19. Dessa forma, mais pesquisas devem ser realizadas nesta linha de investigação a fim de comprovar a importância da farinha para a prevenção e/ou tratamento efetivo da Covid-19.

\section{Referências}

Almeida, R. R., Lacerda, L. G., Murakami, F. S., Bannach, G., Demiate, I. M., Soccol, C. R., \& Schnitzler, E. (2011). Thermal analysis as a screening technique for the characterization of babassu flour and its solid fractions after acid and enzymatic hydrolysis. Thermochimica Acta, 519(1-2): 50-54.

Associação Brasileira de Nutrição. (2020). Guia para uma alimentação saudável em tempos de Covid-19. 2020. ASBRAN.

Baldez, R. N., Malafaia, O., Czeczko, N. G., Martin, N. L. P., Ferreira, L. M; Ribas C. A. P. M., \& Araújo, L. R. R. (2017). Análise da cicatrização do cólon com uso do extrato aquoso da Orbignya phalerata (Babaçu) em ratos. Revista Acta Cirúrgica Brasileira, 21: 31-38. 
Barros, I. C. (2011). Avaliação Biofarmacotecnica de potencial excipiente farmaceutico: pó de mesocarpo de babaçu (Orbignya phalerata Mart.) (Dissertação de Mestrado) - Universidade Federal do Piauí, Teresina, PI, Brasil.

Cardoso, L. O., Carvalho, M. S., Cruz, O. G., Melere, C., Luft, V. C., Molina, M. C. B., \& Chor, D. (2016). Eating patterns in the Brazilian Longitudinal Study of Adult Health (ELSA-Brasil): an exploratory analysis. Cadernos de Saúde Pública, 32(5), e00066215.

Cavalcante Neto, A. A. (2012). Desenvolvimento de Massa Alimentícia Mista de Farinhas de Trigo e Mesocarpo de Babaçu (Orbignya sp.) (Dissertação de Mestrado) - Universidade Federal Rural do Rio de Janeiro, RJ, Brasil.

Conselho Federal de Nutricionistas. (2020). Boas práticas para atuação do nutricionista e do técnico em nutrição e dietética durante a pandemia de coronavírus. São Paulo: CFN.

European Food Safety Authority. (2020). Coronavirus: no evidence that food is a source or transmission route. Parma: EFESA.

Garófolo, A., Qiao, L., \& Maia-Lemos, P. S. (2020). Approach to Nutrition in Cancer Patients in the Context of the Coronavirus Disease 2019 (COVID-19) Pandemic: perspectives. Nutrition And Cancer, 22(5):1-9.

Grant, W. B., Lahore, H., Mcdonnell, S. L., Baggerly, C. A., French, C. B., Aliano, J. L., \& Bhattoa, H. P. (2020). Evidence that Vitamin D Supplementation Could Reduce Risk of Influenza and COVID-19 Infections and Deaths. Nutrients, 12(4): 988.

Khalifa, I., Zhu, W., Mohammed, H. H. H., Dutta, K., \& Li, C. (2020). Tannins inhibit SARS-CoV-2 through binding with catalytic dyad residues of 3CL pro: An in silico approach with 19 structural different hydrolysable tannins. Journal of Food Biochemistry. e13432.

Levy, R. B., Claro, R. M., Bandoni, D. H., Mondini, L., \& Monteiro, C. A. (2012). Availability of added sugars in Brazil: distribution, food sources and time trends. Revista Brasileira de Epidemiologia, 15(1), 3-12.

Lima, V. W. S., Azevedo, A. L. S., Nunes, D. G., Fernandes, M. B. C. C., Bento, C. T., Campos, S. D., \& Uehara, S. K. (2020). Doença renal crônica: cuidados com a alimentação e a saúde mental no contexto da Covid-19. Rio de Janeiro: UFRJ; HUCFF; Serviço de Nutrição e Dietética.

Melo, J. H. G, et al. (2007). Análises físico-químicas do pão enriquecido com mesocarpo de babaçu. Anais do II Congresso de Pesquisa e Inovação da Rede Norte Nordeste de Educação Tecnológica, João Pessoa, PB, Brasil.

Ministério do Desenvolvimento Social e Combate à Fome. (2009). Promoção Nacional da Cadeia de Valor do Coco Babaçu. Brasília: MDS; MDA; MMA.

Morais, A. H. A., Maia, J. K. S., Damasceno, K. S. F. S. C., Seabra, L. M.’ A. J., \& Passos, T. S. (2020). Orientações nutricionais para o enfrentamento do COVID-19. Natal: Universidade Federal do Rio Grande do Norte.

Núcleo de Estudos e pesquisas em Alimentação. (2011). Tabela brasileira de composição de alimentos. (4a ed.), NEPA; UNICAMP.

Oliveira, S.P., \& Reyes, F. G. R. (1990). Biscoito com alto teor de fibra de milho: preparo, caracterização química e tecnológica e teste de aceitabilidade. Ciência e Tecnologia de Alimentos, 10(2): 273-86.

Oliveira, T. C., Abranches, M. V., \& Lana, R. M. (2020). Food (in) security in Brazil in the context of the SARS-CoV-2 pandemic. Cadernos de Saúde Pública, 36(4): e00055220.

Organização Mundial de Saúde. (2020). Coronavirus disease 2019 (COVID-19). Geneva: OMS

Organização Pan-Americana da Saúde. (2019). Folha Informativa Alimentação Saudável. Brasília: OPAS.

Pinto, A., Amaral, P., Gaia, C., \& Oliveira, W. (2010). Boas Práticas para Manejo Florestal e Agroindustrial - Produtos Florestais Não Madeireiros: açaí, andiroba, babaçu, castanha do Brasil, copaíba e unha de gato. Belém: Imazon; SEBRAE.

Silva, A. P. S. (2011). Caracterização fisico-quimica e toxicologica do pó de mesocarpo do babaçu (Orbignya phalerata Mart): subsidio para o desenvolvimento de produtos (Dissertação de Mestrado) - Universidade Federal do Piauí, Teresina, PI, Brasil.

Silva, K. G. S., Batista, I. V., Costa, T. T., Simplicio, A. P. M., Landim, L. A. S. R., \& Gomes, F. O. (2017). Propriedades Funcionais do Coco Babaçu, Uma Revisão. Nutrição em pauta, 37: 39-42.

Silva, N. R., Ferreira, A. C. H., Faturi, C., Silva, G. F., Missio, R. L., Neiva, J. N. M., \& Alexandrino, E. (2012). Desempenho em confinamento de bovino de corte, castrados ou não, alimentados com teores crescentes de farelo do mesocarpo de babaçu. Revista Ciência Rural, 42 (10): $1882-1887$.

Souza, A. M., Barufaldi, L. A., Abreu, G. A., Giannini, D. T., Oliveira, C. L., Santos, M. M., \& Vasconcelos, F. A. G. (2016). ERICA: intake of macro and micronutrients of brazilian adolescents. Revista de Saúde Pública, 50(1): 1-15.

Zaitune, M. P. A., Barros, M. B. A., Lima, M. G., César, C. L. G., Carandina, L., Goldbaum, M., \& Alves, M. C. G. P. (2012). Fatores associados ao tabagismo em idosos: Inquérito de Saúde no Estado de São Paulo (ISA-SP). Cadernos de Saúde Pública, 28(3), 583-596. 